\title{
ON APPROACH TO INCREASE DENSITY OF FIELD- EFFECT TRANSISTORS IN AN INVERTER CIRCUIT
}

\author{
E.L. Pankratov and E.A. Bulaeva \\ Nizhny Novgorod State University, 23 Gagarin avenue, Nizhny Novgorod, 603950, \\ Russia
}

\begin{abstract}
In this paper we consider an approach to decrease dimensions of field-effect transistors framework invertors with increasing of their density. Framework the approach it is necessary to manufacture a heterostructure, which consist of two layers. One of them includes into itself several sections. After manufacturing of the heterostructure these sections should be doped by diffusion or by ion implantation with future optimized annealing of dopant and/or radiation defects. To prognosis the technological process we consider an analytical approach, which gives a possibility to take into account variation of physical parameters in space and time. At the same time the approach gives a possibility to take into account nonlinearity of mass and heat transport and to analyze the above transport without crosslinking solutions on interfaces between materials of heterostructure.
\end{abstract}

\section{KEYWORDS}

Field-effect heterotransystors, inverters dynamic and resistive loads, decreasing of dimensions, optimization of manufacturing.

\section{INTRODUCTION}

Intensive development of solid state electronics gives a possibility to increase density of elements of integrated circuits, reliability and performances of their elements (diodes, field-effect and bipolar transistors, et al) and their discrete analogs. Increasing of density of elements of integrated circuits leads to necessity to decrease their dimensions. One way to decrease dimensions of elements of integrated circuits is their manufacturing framework thin film heterostructures [1-4]. An alternative approach to decrease their dimensions is using laser and microwave types of annealing [5-7]. Both types of annealing gives a possibility to generate inhomogenous distribution of temperature. In this situation one can obtain inhomogenous distribution of diffusion coefficients of dopants and radiation defects and other parameters. The inhomogeneity gives a possibility to decrease dimensions of elements of integrated circuits. Properties of electronic materials could be also changed by radiation processing [8,9].

In the present paper we consider circuits of invertors with dynamic and resistive loads [10] (see Fig. 1). Based on recently formulated recommendations to decrease dimensions of single transistors (both bipolar and field-effect) [11-16] we formulate recommendations to increase density of field-effect transistors framework the considered circuits. We assume, that the considered circuits were manufactured framework the considered in Fig.1 heterostructure. The heterostructure consist of a substrate and an epitaxial layer. The epitaxial layer includes into itself several sections, manufactured by using other materials. These sections were doped by diffusion or by ion implantation to generate required types of conductivity $(n$ or $p)$ in the required materials. Framework this paper we analyzed redistribution of dopants during annealing of these dopants and/or radiation defects to formulate conditions, which correspond to decreasing of the considered circuits.

DOI: $10.5121 /$ antj.2017.3201 


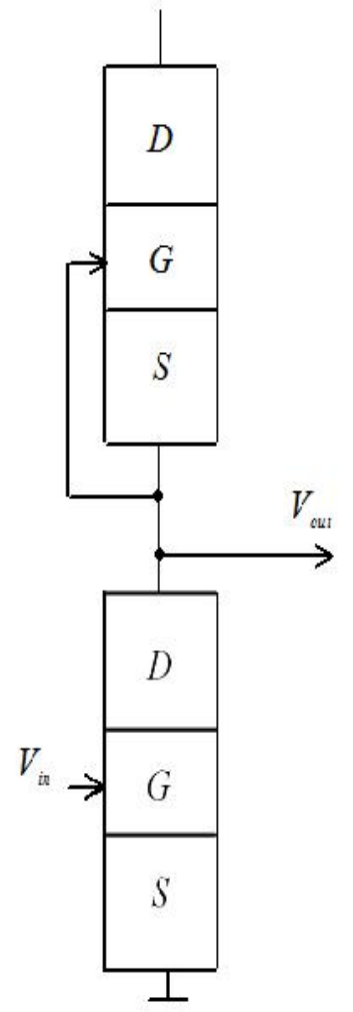

Fig. 1a. Structure of Inverter With Dynamic Load. View from top

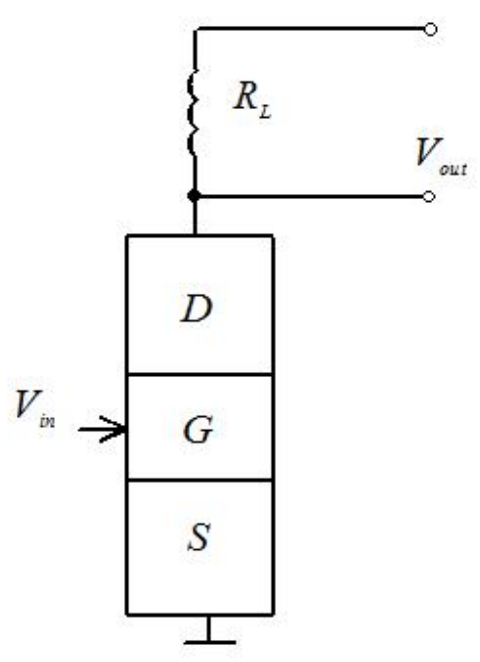

Fig. 1b. Structure of inverter with resistive load. View From Top

\section{Method of Solution}

To solve our aim we shall analyze spatio-temporal distribution of concentration of dopant. The distribution has been determined by solving the following boundary problem

$$
\frac{\partial C(x, y, z, t)}{\partial t}=\frac{\partial}{\partial x}\left[D_{C} \frac{\partial C(x, y, z, t)}{\partial x}\right]+\frac{\partial}{\partial y}\left[D_{C} \frac{\partial C(x, y, z, t)}{\partial y}\right]+\frac{\partial}{\partial z}\left[D_{C} \frac{\partial C(x, y, z, t)}{\partial z}\right] .
$$

Boundary and initial conditions for the equations are

$$
\begin{gathered}
\left.\frac{\partial C(x, y, z, t)}{\partial x}\right|_{x=0}=0,\left.\frac{\partial C(x, y, z, t)}{\partial x}\right|_{x=L_{x}}=0,\left.\frac{\partial C(x, y, z, t)}{\partial y}\right|_{y=0}=0,\left.\frac{\partial C(x, y, z, t)}{\partial y}\right|_{x=L_{y}}=0, \\
\left.\frac{\partial C(x, y, z, t)}{\partial z}\right|_{z=0}=0,\left.\frac{\partial C(x, y, z, t)}{\partial z}\right|_{x=L_{z}}=0, C(x, y, z, 0)=f(x, y, z) .
\end{gathered}
$$

Function $C(x, y, z, t)$ describes the spatio-temporal distribution of concentration of dopant; $T$ is the temperature of annealing; $D_{\mathrm{C}}$ is the dopant diffusion coefficient. Value of dopant diffusion coefficient will be different in different materials and will be changed with changing of temperature of annealing (with account Arrhenius law). The value also depends on concentrations of dopant and radiation defects. All above dependences could be accounted by the following relation $[9,17,18]$

$$
D_{C}=D_{L}(x, y, z, T)\left[1+\xi \frac{C^{\gamma}(x, y, z, t)}{P^{\gamma}(x, y, z, T)}\right]\left[1+\varsigma_{1} \frac{V(x, y, z, t)}{V^{*}}+\varsigma_{2} \frac{V^{2}(x, y, z, t)}{\left(V^{*}\right)^{2}}\right] .(3)
$$


The function $D_{L}(x, y, z, T)$ gives a possibility to take into account the spatial and temperature dependences of dopant diffusion coefficient (due to presents several layers in heterostructure and Arrhenius law). The function $P(x, y, z, T)$ describes the limit of solubility of dopant. The parameter $\gamma \in[1,3]$ describes quantity of charged defects, which were interacted (in average) with atoms of dopant [17]. The function $V(x, y, z, t)$ describes the spatio-temporal distribution of concentration of radiation vacancies. The parameter $V^{*}$ describes the equilibrium distribution of concentration of vacancies. It should be noted, that using diffusion type of doping did not generation radiation defects. In this situation $\zeta_{1}=\zeta_{2}=0$. We determine spatio-temporal distributions of concentrations of radiation defects by solving the following system of equations $[9,18]$

$$
\begin{aligned}
& \frac{\partial I(x, y, z, t)}{\partial t}=\frac{\partial}{\partial x}\left[D_{I}(x, y, z, T) \frac{\partial I(x, y, z, t)}{\partial x}\right]+\frac{\partial}{\partial y}\left[D_{I}(x, y, z, T) \frac{\partial I(x, y, z, t)}{\partial y}\right]-k_{I, I}(x, y, z, T) \times \\
& \quad \times I^{2}(x, y, z, t)+\frac{\partial}{\partial z}\left[D_{I}(x, y, z, T) \frac{\partial I(x, y, z, t)}{\partial z}\right]-k_{I, V}(x, y, z, T) I(x, y, z, t) V(x, y, z, t) \quad(4) \\
& \frac{\partial V(x, y, z, t)}{\partial t}=\frac{\partial}{\partial x}\left[D_{V}(x, y, z, T) \frac{\partial V(x, y, z, t)}{\partial x}\right]+\frac{\partial}{\partial y}\left[D_{V}(x, y, z, T) \frac{\partial V(x, y, z, t)}{\partial y}\right]-k_{V, V}(x, y, z, T) \times \\
& \times V^{2}(x, y, z, t)+\frac{\partial}{\partial z}\left[D_{V}(x, y, z, T) \frac{\partial V(x, y, z, t)}{\partial z}\right]-k_{I, V}(x, y, z, T) I(x, y, z, t) V(x, y, z, t) .
\end{aligned}
$$

Boundary and initial conditions for these equations are

$$
\begin{gathered}
\left.\frac{\partial \rho(x, y, z, t)}{\partial x}\right|_{x=0}=0,\left.\frac{\partial \rho(x, y, z, t)}{\partial x}\right|_{x=L_{x}}=0,\left.\frac{\partial \rho(x, y, z, t)}{\partial y}\right|_{y=0}=0,\left.\frac{\partial \rho(x, y, z, t)}{\partial y}\right|_{y=L_{y}}=0, \\
\left.\frac{\partial \rho(x, y, z, t)}{\partial z}\right|_{z=0}=0,\left.\frac{\partial \rho(x, y, z, t)}{\partial z}\right|_{z=L_{z}}=0, \rho(x, y, z, 0)=f_{\rho}(x, y, z) .
\end{gathered}
$$

Here $\rho=I, V$. The function $I(x, y, z, t)$ describes variation of distribution of concentration of radiation interstitials in space and time. The function $D_{\rho}(x, y, z, T)$ describes dependences of the diffusion coefficients of point radiation defects on spatial coordinates and temperature. Terms $V^{2}(x, y, z, t)$ and $I^{2}(x, y, z, t)$ correspond to generation divacancies and diinterstitials; $k_{I, V}(x, y, z, T)$ is the parameter of recombination of point radiation defects; $k_{I, I}(x, y, z, T)$ and $k_{V, V}(x, y, z, T)$ are the parameters of generation of simplest complexes of point radiation defects.

We determine concentrations of divacancies $\Phi_{V}(x, y, z, t)$ and dinterstitials $\Phi_{I}(x, y, z, t)$ as functions of space and time by solving the following system of equations $[17,18]$

$$
\begin{aligned}
& \frac{\partial \Phi_{I}(x, y, z, t)}{\partial t}=\frac{\partial}{\partial x}\left[D_{\Phi I}(x, y, z, T) \frac{\partial \Phi_{I}(x, y, z, t)}{\partial x}\right]+\frac{\partial}{\partial y}\left[D_{\Phi I}(x, y, z, T) \frac{\partial \Phi_{I}(x, y, z, t)}{\partial y}\right]+ \\
& +\frac{\partial}{\partial z}\left[D_{\Phi I}(x, y, z, T) \frac{\partial \Phi_{I}(x, y, z, t)}{\partial z}\right]+k_{I, I}(x, y, z, T) I^{2}(x, y, z, t)-k_{I}(x, y, z, T) I(x, y, z, t) \\
& \frac{\partial \Phi_{V}(x, y, z, t)}{\partial t}=\frac{\partial}{\partial x}\left[D_{\Phi V}(x, y, z, T) \frac{\partial \Phi_{V}(x, y, z, t)}{\partial x}\right]+\frac{\partial}{\partial y}\left[D_{\Phi V}(x, y, z, T) \frac{\partial \Phi_{V}(x, y, z, t)}{\partial y}\right]+ \\
& +\frac{\partial}{\partial z}\left[D_{\Phi V}(x, y, z, T) \frac{\partial \Phi_{V}(x, y, z, t)}{\partial z}\right]+k_{V, V}(x, y, z, T) V^{2}(x, y, z, t)-k_{V}(x, y, z, T) V(x, y, z, t) .
\end{aligned}
$$


Boundary and initial conditions for these equations are

$$
\begin{gathered}
\left.\frac{\partial \Phi_{\rho}(x, y, z, t)}{\partial x}\right|_{x=0}=0,\left.\frac{\partial \Phi_{\rho}(x, y, z, t)}{\partial x}\right|_{x=L_{x}}=0,\left.\frac{\partial \Phi_{\rho}(x, y, z, t)}{\partial y}\right|_{y=0}=0,\left.\frac{\partial \Phi_{\rho}(x, y, z, t)}{\partial y}\right|_{y=L_{y}}=0, \\
\left.\frac{\partial \Phi_{\rho}(x, y, z, t)}{\partial z}\right|_{z=0}=0,\left.\frac{\partial \Phi_{\rho}(x, y, z, t)}{\partial z}\right|_{z=L_{z}}=0, \Phi_{I}(x, y, z, 0)=f_{\Phi I}(x, y, z), \Phi_{V}(x, y, z, 0)=f_{\Phi V}(x, y, z) .(7)
\end{gathered}
$$

Here $D_{\Phi \rho}(x, y, z, T)$ are the diffusion coefficients of the above complexes of radiation defects; $k_{I}(x, y, z, T)$ and $k_{V}(x, y, z, T)$ are the parameters of decay of these complexes.

We determine spatio-temporal distributions of concentrations of dopant and radiation defects by using method of averaging of function corrections [19] with decreased quantity of iteration steps [20]. Framework the approach we used solutions of Eqs. (1), (4) and (6) in linear form and with averaged values of diffusion coefficients $D_{0 L}, D_{0 I}, D_{0 V}, D_{0 \Phi I}, D_{0 \Phi V}$ as initial-order approximations of the required concentrations. The solutions could be written as

$$
\begin{gathered}
C_{1}(x, y, z, t)=\frac{F_{0 C}}{L_{x} L_{y} L_{z}}+\frac{2}{L_{x} L_{y} L_{z}} \sum_{n=1}^{\infty} F_{n C} c_{n}(x) c_{n}(y) c_{n}(z) e_{n C}(t), \\
I_{1}(x, y, z, t)=\frac{F_{0 I}}{L_{x} L_{y} L_{z}}+\frac{2}{L_{x} L_{y} L_{z}} \sum_{n=1}^{\infty} F_{n I} c_{n}(x) c_{n}(y) c_{n}(z) e_{n I}(t), \\
V_{1}(x, y, z, t)=\frac{F_{0 C}}{L_{x} L_{y} L_{z}}+\frac{2}{L_{x} L_{y} L_{z}} \sum_{n=1}^{\infty} F_{n C} c_{n}(x) c_{n}(y) c_{n}(z) e_{n V}(t), \\
\Phi_{I 1}(x, y, z, t)=\frac{F_{0 \Phi_{I}}}{L_{x} L_{y} L_{z}}+\frac{2}{L_{x} L_{y} L_{z}} \sum_{n=1}^{\infty} F_{n \Phi_{I}} c_{n}(x) c_{n}(y) c_{n}(z) e_{n \Phi_{I}}(t), \\
\Phi_{V 1}(x, y, z, t)=\frac{F_{0 \Phi_{V}}}{L_{x} L_{y} L_{z}}+\frac{2}{L_{x} L_{y} L_{z}} \sum_{n=1}^{\infty} F_{n \Phi_{V}} c_{n}(x) c_{n}(y) c_{n}(z) e_{n \Phi_{V}}(t), \\
\text { where } e_{n \rho}(t)=\exp \left[-\pi^{2} n^{2} D_{0 \rho} t\left(\frac{1}{L_{x}^{2}}+\frac{1}{L_{y}^{2}}+\frac{1}{L_{z}^{2}}\right)\right], F_{n p}=\int_{0}^{L_{x}} c_{n}(u) \iint_{0}^{L_{y}} c_{n}(v) \int_{0}^{L_{z}} c_{n}(v) f_{\rho}(u, v, w) d w d v d u, c_{n}(\chi)=
\end{gathered}
$$$$
\cos \left(\pi n \chi / L_{\chi}\right) \text {. }
$$

We consider the above solutions as initial-order approximations of concentrations of dopant and radiation defects.

Approximations of concentrations of dopant and radiation defects with the second and higher orders could be determine framework standard iterative procedure $[19,20]$. The procedure based on replacement of the functions $C(x, y, z, t), I(x, y, z, t), V(x, y, z, t), \Phi_{I}(x, y, z, t), \Phi_{V}(x, y, z, t)$ in the right sides of the Eqs. (1), (4) and (6) on the following sums $\alpha_{n \rho}+\rho_{n-1}(x, y, z, t)$. Framework the standard iterative procedure we obtain equations for the second-order approximations of concentrations of dopant and radiation defects

$$
\frac{\partial C_{2}(x, y, z, t)}{\partial t}=\frac{\partial}{\partial x}\left(\left[1+\varsigma_{1} \frac{V(x, y, z, t)}{V^{*}}+\varsigma_{2} \frac{V^{2}(x, y, z, t)}{\left(V^{*}\right)^{2}}\right]\left\{1+\xi \frac{\left[\alpha_{2 C}+C_{1}(x, y, z, t)\right]^{\gamma}}{P^{\gamma}(x, y, z, T)}\right\} \times\right.
$$


Advanced Nanoscience and Technology: An International Journal (ANTJ), Vol. 3, No.1/2, June 2017

$$
\begin{gathered}
\left.\times D_{L}(x, y, z, T) \frac{\partial C_{1}(x, y, z, t)}{\partial x}\right)+\frac{\partial}{\partial y}\left(D_{L}(x, y, z, T)\left[1+\varsigma_{1} \frac{V(x, y, z, t)}{V^{*}}+\varsigma_{2} \frac{V^{2}(x, y, z, t)}{\left(V^{*}\right)^{2}}\right] \times\right. \\
\left.\times\left\{1+\xi \frac{\left[\alpha_{2 C}+C_{1}(x, y, z, t)\right]^{\gamma}}{P^{\gamma}(x, y, z, T)}\right\} \frac{\partial C_{1}(x, y, z, t)}{\partial y}\right)+\frac{\partial}{\partial z}\left(D_{L}(x, y, z, T) \frac{\partial C_{1}(x, y, z, t)}{\partial z} \times\right. \\
\left.\times\left[1+\varsigma_{1} \frac{V(x, y, z, t)}{V^{*}}+\varsigma_{2} \frac{V^{2}(x, y, z, t)}{\left(V^{*}\right)^{2}}\right]\left\{1+\xi \frac{\left[\alpha_{2 C}+C_{1}(x, y, z, t)\right]^{\gamma}}{P^{\gamma}(x, y, z, T)}\right\}\right)
\end{gathered}
$$

$$
\left\{\begin{array}{l}
\frac{\partial I_{2}(x, y, z, t)}{\partial t}=\frac{\partial}{\partial x}\left[D_{I}(x, y, z, T) \frac{\partial I_{1}(x, y, z, t)}{\partial x}\right]+\frac{\partial}{\partial y}\left[D_{I}(x, y, z, T) \frac{\partial I_{1}(x, y, z, t)}{\partial y}\right]+ \\
+\frac{\partial}{\partial z}\left[D_{I}(x, y, z, T) \frac{\partial I_{1}(x, y, z, t)}{\partial z}\right]-\left[\alpha_{2 I}+I_{1}(x, y, z, t)\right]\left[\alpha_{2 V}+V_{1}(x, y, z, t)\right] \times \\
\times k_{I, V}(x, y, z, T)-k_{I, I}(x, y, z, T)\left[\alpha_{2 I}+I_{1}(x, y, z, t)\right]^{2} \\
\frac{\partial V_{2}(x, y, z, t)}{\partial t}=\frac{\partial}{\partial x}\left[D_{V}(x, y, z, T) \frac{\partial V_{1}(x, y, z, t)}{\partial x}\right]+\frac{\partial}{\partial y}\left[D_{V}(x, y, z, T) \frac{\partial V_{1}(x, y, z, t)}{\partial y}\right]+ \\
+\frac{\partial}{\partial z}\left[D_{V}(x, y, z, T) \frac{\partial V_{1}(x, y, z, t)}{\partial z}\right]-\left[\alpha_{2 I}+I_{1}(x, y, z, t)\right]\left[\alpha_{2 V}+V_{1}(x, y, z, t)\right] \times \\
\times k_{I, V}(x, y, z, T)-k_{V, V}(x, y, z, T)\left[\alpha_{2 V}+V_{1}(x, y, z, t)\right]^{2}
\end{array}\right.
$$

$$
\left\{\begin{array}{l}
\frac{\partial \Phi_{I 2}(x, y, z, t)}{\partial t}=\frac{\partial}{\partial x}\left[D_{\Phi_{I}}(x, y, z, T) \frac{\partial \Phi_{I 1}(x, y, z, t)}{\partial x}\right]+\frac{\partial}{\partial y}\left[D_{\Phi_{I}}(x, y, z, T) \times\right. \\
\left.\times \frac{\partial \Phi_{I 1}(x, y, z, t)}{\partial y}\right]+\frac{\partial}{\partial z}\left[D_{\Phi_{I}}(x, y, z, T) \frac{\partial \Phi_{I 1}(x, y, z, t)}{\partial z}\right]+k_{I, I}(x, y, z, T) \times \\
\times I^{2}(x, y, z, t)-k_{I}(x, y, z, T) I(x, y, z, t) \\
\frac{\partial \Phi_{V 2}(x, y, z, t)}{\partial t}=\frac{\partial}{\partial x}\left[D_{\Phi_{V}}(x, y, z, T) \frac{\partial \Phi_{V 1}(x, y, z, t)}{\partial x}\right]+\frac{\partial}{\partial y}\left[D_{\Phi_{V}}(x, y, z, T) \times\right. \\
\left.\times \frac{\partial \Phi_{I 1}(x, y, z, t)}{\partial y}\right]+\frac{\partial}{\partial z}\left[D_{\Phi_{V}}(x, y, z, T) \frac{\partial \Phi_{V 1}(x, y, z, t)}{\partial z}\right]+k_{V, V}(x, y, z, T) \times \\
\times V^{2}(x, y, z, t)-k_{V}(x, y, z, T) V(x, y, z, t)
\end{array}\right.
$$

Integration of the left and right sides of Eqs.(8)-(10) gives us possibility to obtain relations for the second-order approximations of concentrations of dopant and radiation defects in final form

$$
\begin{aligned}
& C_{2}(x, y, z, t)=\frac{\partial}{\partial x}\left(\int_{0}^{t}\left[1+\varsigma_{1} \frac{V(x, y, z, \tau)}{V^{*}}+\varsigma_{2} \frac{V^{2}(x, y, z, \tau)}{\left(V^{*}\right)^{2}}\right]\left\{1+\xi \frac{\left[\alpha_{2 C}+C_{1}(x, y, z, \tau)\right]^{\gamma}}{P^{\gamma}(x, y, z, T)}\right\} \times\right. \\
& \left.\times D_{L}(x, y, z, T) \frac{\partial C_{1}(x, y, z, \tau)}{\partial x}\right) d \tau+\frac{\partial}{\partial y}\left(\int_{0}^{t}\left[1+\varsigma_{1} \frac{V(x, y, z, \tau)}{V^{*}}+\varsigma_{2} \frac{V^{2}(x, y, z, \tau)}{\left(V^{*}\right)^{2}}\right] \times\right.
\end{aligned}
$$


Advanced Nanoscience and Technology: An International Journal (ANTJ), Vol. 3, No.1/2, June 2017

$$
\begin{aligned}
& \left.\times D_{L}(x, y, z, T)\left\{1+\xi \frac{\left[\alpha_{2 C}+C_{1}(x, y, z, \tau)\right]^{\gamma}}{P^{\gamma}(x, y, z, T)}\right\} \frac{\partial C_{1}(x, y, z, \tau)}{\partial y} d \tau\right)+f_{C}(x, y, z)+\frac{\partial}{\partial z}\left(\int_{0}^{t} D_{L}(x, y, z, T) \times\right. \\
& \left.\times\left[1+\varsigma_{1} \frac{V(x, y, z, \tau)}{V^{*}}+\varsigma_{2} \frac{V^{2}(x, y, z, \tau)}{\left(V^{*}\right)^{2}}\right]\left\{1+\xi \frac{\left[\alpha_{2 C}+C_{1}(x, y, z, \tau)\right]^{\gamma}}{P^{\gamma}(x, y, z, T)}\right\} \frac{\partial C_{1}(x, y, z, \tau)}{\partial z} d \tau\right)(8 a) \\
& I_{2}(x, y, z, t)=\frac{\partial}{\partial x}\left[\int_{0}^{t} D_{I}(x, y, z, T) \frac{\partial I_{1}(x, y, z, \tau)}{\partial x} d \tau\right]+\frac{\partial}{\partial y}\left[\int_{0}^{t} D_{I}(x, y, z, T) \frac{\partial I_{1}(x, y, z, \tau)}{\partial y} d \tau\right]+ \\
& +\frac{\partial}{\partial z}\left[\int_{0}^{t} D_{I}(x, y, z, T) \frac{\partial I_{1}(x, y, z, \tau)}{\partial z} d \tau\right]-\int_{0}^{t} k_{I, I}(x, y, z, T)\left[\alpha_{2 I}+I_{1}(x, y, z, \tau)\right]^{2} d \tau+ \\
& +f_{I}(x, y, z)-\int_{0}^{t} k_{I, V}(x, y, z, T)\left[\alpha_{2 I}+I_{1}(x, y, z, \tau)\right]\left[\alpha_{2 V}+V_{1}(x, y, z, \tau)\right] d \tau \\
& V_{2}(x, y, z, t)=\frac{\partial}{\partial x}\left[\int_{0}^{t} D_{V}(x, y, z, T) \frac{\partial V_{1}(x, y, z, \tau)}{\partial x} d \tau\right]+\frac{\partial}{\partial y}\left[\int_{0}^{t} D_{V}(x, y, z, T) \frac{\partial V_{1}(x, y, z, \tau)}{\partial y} d \tau\right]+ \\
& \left.\times \frac{\partial V_{1}(x, y, z, \tau)}{\partial y} d \tau\right]+\frac{\partial}{\partial z}\left[\int_{0}^{t} D_{V}(x, y, z, T) \frac{\partial V_{1}(x, y, z, \tau)}{\partial z} d \tau\right]-\int_{0}^{t} k_{V, V}(x, y, z, T)\left[\alpha_{2 I}+V_{1}(x, y, z, \tau)\right]^{2} d \tau+ \\
& +f_{V}(x, y, z)-\int_{0}^{t} k_{I, V}(x, y, z, T)\left[\alpha_{2 I}+I_{1}(x, y, z, \tau)\right]\left[\alpha_{2 V}+V_{1}(x, y, z, \tau)\right] d \tau \\
& \Phi_{I 2}(x, y, z, t)=\frac{\partial}{\partial x}\left[\int_{0}^{t} D_{\Phi_{I}}(x, y, z, T) \frac{\partial \Phi_{I 1}(x, y, z, \tau)}{\partial x} d \tau\right]+\frac{\partial}{\partial y}\left[\int_{0}^{t} D_{\Phi_{I}}(x, y, z, T) \frac{\partial \Phi_{I 1}(x, y, z, \tau)}{\partial y} d \tau\right]+ \\
& +\frac{\partial}{\partial z}\left[\int_{0}^{t} D_{\Phi_{I}}(x, y, z, T) \frac{\partial \Phi_{I 1}(x, y, z, \tau)}{\partial z} d \tau\right]+\int_{0}^{t} k_{I, I}(x, y, z, T) I^{2}(x, y, z, \tau) d \tau+ \\
& +f_{\Phi_{I}}(x, y, z)-\int_{0}^{t} k_{I}(x, y, z, T) I(x, y, z, \tau) d \tau \\
& \Phi_{V 2}(x, y, z, t)=\frac{\partial}{\partial x}\left[\int_{0}^{t} D_{\Phi_{V}}(x, y, z, T) \frac{\partial \Phi_{V 1}(x, y, z, \tau)}{\partial x} d \tau\right]+\frac{\partial}{\partial y}\left[\int_{0}^{t} D_{\Phi_{V}}(x, y, z, T) \frac{\partial \Phi_{V 1}(x, y, z, \tau)}{\partial y} d \tau\right]+ \\
& +\frac{\partial}{\partial z}\left[\int_{0}^{t} D_{\Phi_{V}}(x, y, z, T) \frac{\partial \Phi_{V 1}(x, y, z, \tau)}{\partial z} d \tau\right]+\int_{0}^{t} k_{V, V}(x, y, z, T) V^{2}(x, y, z, \tau) d \tau+ \\
& +f_{\Phi_{V}}(x, y, z)-\int_{0}^{t} k_{V}(x, y, z, T) V(x, y, z, \tau) d \tau
\end{aligned}
$$

Average values of the considered approximations have been determined by the following relations $[19,20]$

$$
\alpha_{2 \rho}=\frac{1}{\Theta L_{x} L_{y} L_{z}} \int_{0}^{\Theta} \int_{0}^{L_{x} L_{y} L_{2}} \int_{0}^{0}\left[\rho_{0}(x, y, z, t)-\rho_{1}(x, y, z, t)\right] d z d y d x d t
$$

Substitution of approximations $(8 a)-(10 a)$ into the previous relation gives the possibility to obtain relations for the average values $\alpha_{2 \rho}$ in the following final form 
Advanced Nanoscience and Technology: An International Journal (ANTJ), Vol. 3, No.1/2, June 2017

$$
\begin{gathered}
\alpha_{2 C}=\frac{1}{L_{x} L_{y} L_{z}} \int_{0}^{L_{x} L_{y} L_{z}} \int_{0} f_{C}(x, y, z) d z d y d x \\
\alpha_{2 I}=\frac{1}{2 A_{I I 00}}\left\{\left(1+A_{I V 01}+A_{I I 10}+\alpha_{2 V} A_{I V 00}\right)^{2}-4 A_{I I 00}\left[\alpha_{2 V} A_{I V 10}-A_{I I 20}+A_{I V 11}-\right.\right. \\
\left.\left.-\frac{1}{L_{x} L_{y} L_{z}} \int_{0}^{L_{x} L_{y} L_{z}} \int_{0}^{z} f_{I}(x, y, z) d z d y d x\right]\right\}^{\frac{1}{2}}-\frac{1+A_{I V 01}+A_{I I 10}+\alpha_{2 V} A_{I V 00}}{2 A_{I I 00}} \\
\alpha_{2 V}=\frac{1}{2 B_{4}} \sqrt{\frac{\left(B_{3}+A\right)^{2}}{4}-4 B_{4}\left(y+\frac{B_{3} y-B_{1}}{A}\right)}-\frac{B_{3}+A}{4 B_{4}}
\end{gathered}
$$

Here $A_{a b i j}=\frac{1}{\Theta L_{x} L_{y} L_{z}} \int_{0}^{\Theta}(\Theta-t) \int_{0}^{L_{L} L_{y}} \int_{0}^{L_{z}} \int_{0} k_{a, b}(x, y, z, T) I_{1}^{i}(x, y, z, t) V_{1}^{j}(x, y, z, t) d z d y d x d t, B_{4}=A_{I V 00}^{2} A_{I V 00}^{2}-$ $-2\left(A_{I V 00}^{2}-A_{I I 00} A_{V V 00}\right)^{2}, B_{3}=A_{I V 00} A_{I V 00}^{2}+A_{I V 01} A_{I V 00}^{3}+A_{I V 00} A_{I I 10} A_{I V 00}^{2}-4\left(A_{I V 00}^{2}-A_{I I 00} A_{V V 00}\right)\left[A_{I V 01} \times\right.$ $\left.\times 2 A_{I V 00}+2 A_{I V 00}\left(1+A_{I V 01}+A_{I I 10}\right)-2 A_{I I 00}\left(A_{I V 10}+A_{V V 10}+1\right)\right]-4 A_{I V 10} A_{I I 00} A_{I V 00}^{2}+2 A_{I V 00} A_{I V 01} A_{I V 00}^{2}$, $B_{2}=A_{I V 00}^{2}\left\{\left(1+A_{I V 01}+A_{I I 10}\right)^{2}+A_{I V 00}^{2} A_{I V 01}^{2}+2 A_{I V 00} A_{I V 00}\left(A_{I V 00}+A_{I V 00} A_{I V 01}+A_{I V 00} A_{I I 10}-4 A_{I V 10} A_{I I 00}\right)-\right.$ $-4 A_{I I 00}\left[A_{I V 11}-A_{I I 20}-\frac{1}{L_{x} L_{y} L_{z}} \int_{0}^{L_{x}} \int_{0}^{L_{y}} \int_{0}^{L_{z}} f_{I}(x, y, z) d z d y d x\right]\left\{\left\{2 A_{I V 01} A_{I V 00}+2 A_{I V 00}\left(1+A_{I V 01}+A_{I I 10}\right)-\right.\right.$ $\left.-2 A_{I I 00}\left(A_{I V 10}+1+A_{V V 10}\right)\right]^{2}+2\left[A_{I V 01}\left(1+A_{I V 01}+A_{I I 10}\right)+\frac{2}{L_{x} L_{y} L_{z}} \int_{0}^{L_{x}} \int_{0}^{L_{y} L_{z}} \int_{0} f_{V}(x, y, z) d z d y d x-2 A_{I I 00} \times\right.$ $\left.\times\left(A_{V V 20}-A_{I V 11}\right)+A_{I V 01}\left(1+A_{I V 01}+A_{I I 10}\right)\right]\left[2 A_{I V 00}\left(1+A_{I V 01}+A_{I I 10}\right)+2 A_{I V 01} A_{I V 00}-2\left(A_{I V 10}+1+A_{V V 10}\right) \times\right.$ $\left.\left.\times A_{I I 00}\right]\right\}, B_{1}=2 A_{I V 00} A_{I V 01}\left(1+A_{I V 01}+A_{I I 10}\right)^{2}-8\left[A_{I V 11}-A_{I I 20}-\frac{1}{L_{x} L_{y} L_{z}} \int_{0}^{L_{x}} \int_{0}^{L_{y}} \int_{0}^{L_{z}} f_{I}(x, y, z) d z d y d x\right]+$ $+A_{I V 00} A_{I V 01} A_{I I 00}+A_{I V 01}^{2}\left(A_{I V 00}+A_{I V 00} A_{I V 01}+A_{I V 00} A_{I I 10}-4 A_{I V 10} A_{I I 00}\right)-2\left[A_{I V 01}\left(1+A_{I V 01}+A_{I I 10}\right)+\right.$ $\left.+\frac{2 A_{I I 00}}{L_{x} L_{y} L_{z}} \int_{0}^{L_{x}} \int_{0}^{L_{y}} \int_{0}^{L_{z}} f_{I}(x, y, z) d z d y d x-2 A_{I I 00}\left(A_{V V 20}-A_{I V 11}\right)+A_{I V 01}\left(1+A_{I V 01}+A_{I I 10}\right)\right]\left[2 A_{I V 00}\left(1+A_{I V 01}+\right.\right.$ $\left.\left.+A_{I I 10}\right)-2\left(A_{I V 10}+A_{V V 10}+1\right) A_{I I 00}+2 A_{I V 01} A_{I V 00}\right], B_{0}=4\left[\frac{1}{L_{x} L_{y} L_{z}} \int_{0}^{L_{x}} \int_{0}^{L_{y}} \int_{0}^{L_{z}} f_{I}(x, y, z) d z d y d x-A_{I V 11}+\right.$ $\left.+A_{I I 20}\right] A_{I I 00} A_{I V 01}^{2}+A_{I V 01}^{2}\left(A_{I V 01}+A_{I I 10}+1\right)^{2}-\left[A_{I V 01}\left(1+A_{I V 01}+A_{I I 10}\right)-2 A_{I I 00}\left(A_{V V 20}-A_{I V 11}\right)+A_{I V 01} \times\right.$ $\left.\times\left(1+A_{I V 01}+A_{I I 0}\right)+\frac{2 A_{I I 00}}{L_{x} L_{y} L_{z}} \int_{0}^{L_{x}} \int_{0}^{L_{y} L_{z}} \int_{0} f_{V}(x, y, z) d z d y d x\right]^{2}, y=\sqrt[3]{\sqrt{q^{2}+p^{3}}-q}-\sqrt[3]{\sqrt{q^{2}+p^{3}}+q}+\frac{B_{2}}{6}$, $q=\left(2 B_{1} B_{3}-8 B_{0}\right) \frac{B_{2}}{48}+\frac{B_{2}^{3}}{216}+\frac{B_{0}\left(4 B_{2}-B_{3}^{2}\right)-B_{1}^{2}}{8}, p=\frac{1}{72}\left[3\left(2 B_{1} B_{3}-8 B_{0}\right)-2 B_{2}^{2}\right], A=\sqrt{8 y+B_{3}^{2}-4 B_{2}}$, $\alpha_{2 \Phi_{I}}=A_{I I 20}-\frac{1}{\Theta L_{x} L_{y} L_{z}} \int_{0}^{\Theta}(\Theta-t) \int_{0}^{L_{x} L_{y} L_{0} \int_{0}} k_{0}(x, y, z, T) I(x, y, z, t) d z d y d x d t+$ $+\frac{1}{L_{x} L_{y} L_{z}} \int_{0}^{L_{x} L_{y} L_{z}} \int_{0} \int_{0}^{z} f_{\Phi I}(x, y, z) d z d y d x$ 


$$
\begin{aligned}
\alpha_{2 \Phi_{V}}=A_{V V 20}-\frac{1}{\Theta L_{x} L_{y} L_{z}} \int_{0}^{\Theta}(\Theta-t) \int_{0}^{L_{x}} \int_{0}^{L_{y} L_{z}} \int_{0} k_{V}(x, y, z, T) V(x, y, z, t) d z d y d x d t+ \\
+\frac{1}{L_{x} L_{y} L_{z}} \int_{0}^{L_{x}} \int_{0}^{L_{y} L_{z}} \int_{0} f_{\Phi V}(x, y, z) d z d y d x .
\end{aligned}
$$

After the substitution we obtain the equation for parameter $\alpha_{2 C}$ for any value of parameter $\gamma$. We analyzed distributions of concentrations of dopant and radiation defects in space and time by using the second-order approximations framework the method of averaged of function corrections. The obtained analytical results have been checked by comparison with results of numerical simulation.

\section{DISCUSSION}

In this section we analyzed variation of concentration of dopant in space and time with account spatio-temporal distribution of concentration of radiation defects. Typical spatial distributions of concentrations of dopants in heterostructures are presented in Figs. 2 and 3. The Fig. 2 shows spatial distributions of infused dopant in direction, which is perpendicular to the interface between materials of the heterostructure for fixed value of annealing time. Increasing of number of curves corresponds to increasing of difference between values of dopant diffusion coefficients of materials of the heterostructure for the case, when dopant diffusion coefficient in doped material is larger, than in nearest material. Fig. 3 shows spatial distributions of concentrations of implanted dopant in direction, which is perpendicular to the interface between materials of the heterostructure. Curves 1 and 3 corresponds to annealing time $\Theta=0,0048\left(L_{x}{ }^{2}+L_{y}{ }^{2}+L_{z}{ }^{2}\right) / D_{0}$. Curves 2 and 4 corresponds to annealing time $\Theta=0,0057\left(L_{x}{ }^{2}+L_{y}{ }^{2}+L_{z}{ }^{2}\right) / D_{0}$ (кривые 2 и 4). Curves 1 and 2 are calculated distributions of concentration of dopant in homogenous material with averaged diffusion coefficient of dopant. Curves 3 and 4 are calculated distributions of concentration of dopant in the considered heterostructure, when dopant diffusion coefficient in doped material is larger, than in nearest material. The figures show, that inhomogeneity of heterostructure gives a possibility to manufacture more compact distribution of concentration of dopant in enriched area. At the same time one can find increasing of homogeneity of the above distribution.

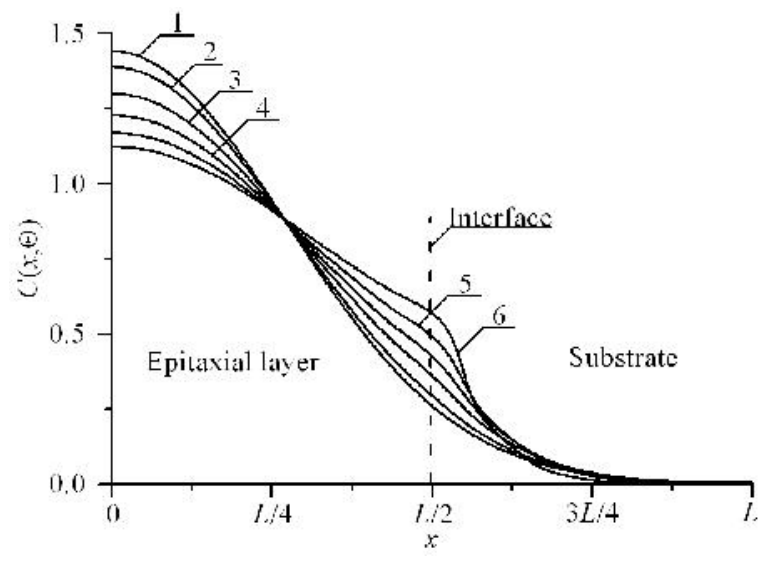

Fig. 2. Spatial distributions of concentration of infused dopant in heterostructure from Fig. 1 in direction, which is perpendicular to interface between materials 


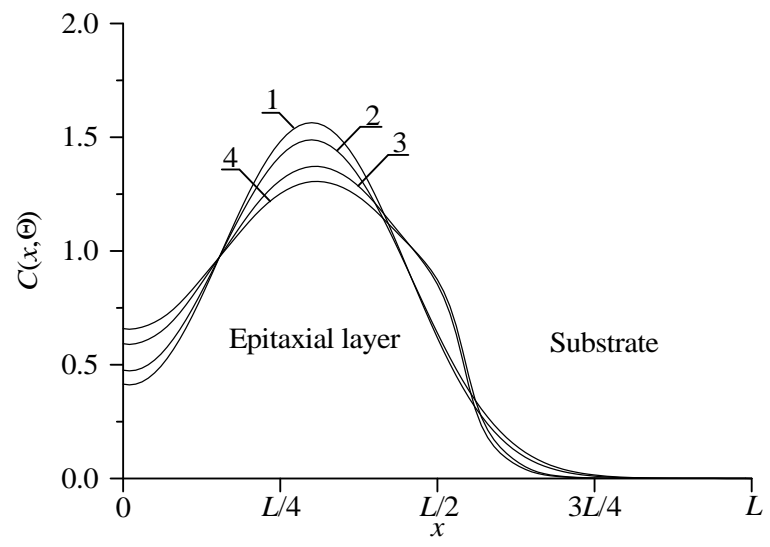

Fig. 3. Spatial distributions of concentration of implanted dopant in heterostructure from Fig. 1 in direction, which is perpendicular to interface between materials

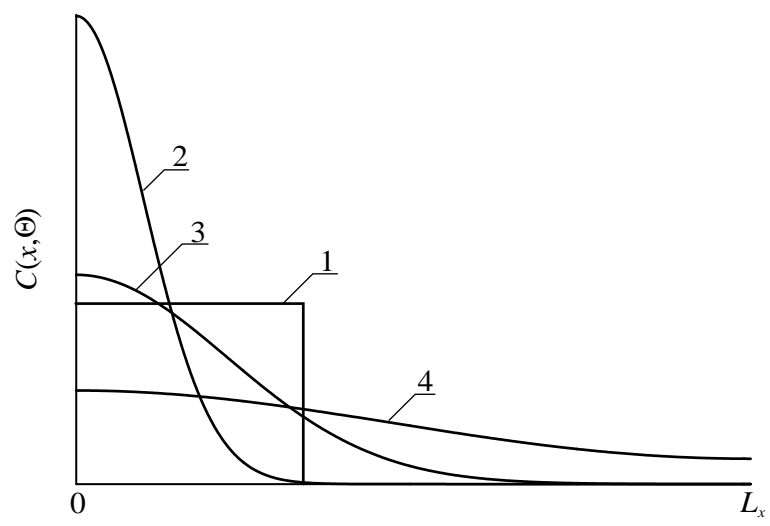

Fig. 4. Spatial distributions of dopant in heterostructure after dopant infusion. Curve 1 is an idealized distribution of dopant. Curves 2-4 are real distributions of dopant for different values of annealing time. The increasing number in the curve corresponds to the increasing annealing time

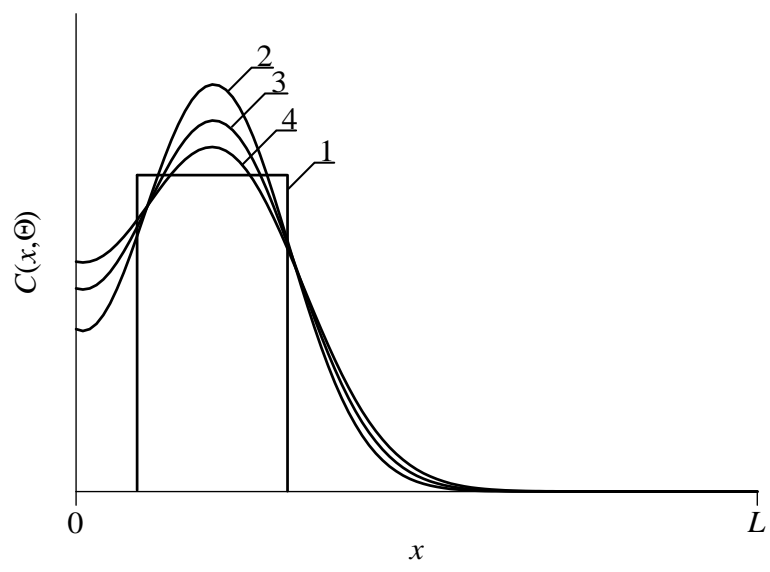

Fig. 5. Spatial distributions of dopant in heterostructure after ion implantation. Curve 1 is an idealized distribution of dopant. Curves 2-4 are real distributions of dopant for different values of annealing time. The increasing number in the curve corresponds to the increasing annealing time 
It should be noted, that framework the considered approach of manufacturing of transistor annealing should be optimized. Reason of the optimization is following. The main reason for this optimization is following. If the annealing time is small, the dopant does not achieves any interfaces between the materials of heterostructure. In this situation one can not find any modifications of the distribution of concentration of dopant. If the annealing time is large, the distribution of concentration of dopant is too homogenous. We optimize the annealing time based on a recently introduced approach [11-16]. By applying the criterion we approximate real distribution of concentration of dopant by a step-wise function (see Figs. 4 and 5). Next we determine optimal values of annealing time by minimization of the following mean-squared error

$$
U=\frac{1}{L_{x} L_{y} L_{z}} \int_{0}^{L_{x} L_{y}} \int_{0}^{L_{z}} \int_{0}[C(x, y, z, t)-\psi(x, y, z)] d z d y d x
$$

where $\psi(x, y, z)$ is the step-wise approximation of spatial distribution of concentration of dopant $C$ $(x, y, z, t)$. We minimize the mean-squared error as the function of time. After the minimization we obtain optimal value of annealing time $\Theta$. Dependences of optimal values of annealing time on parameters are presented in Figs. 6 and 7 for diffusion and ion types of doping, respectively. It should be noted, that it is necessary to anneal radiation defects after ion implantation. One could find spreading of concentration of the distribution of concentration of dopant during this annealing. In the ideal case distribution of dopant achieves appropriate interfaces between materials of heterostructure during annealing of radiation defects. If dopant does not achieve to the nearest interface during annealing of radiation defects, it is practical to additionally anneal the dopant. In this situation the optimal value of the additional annealing time of implanted dopant is smaller, than the annealing time of infused dopant.

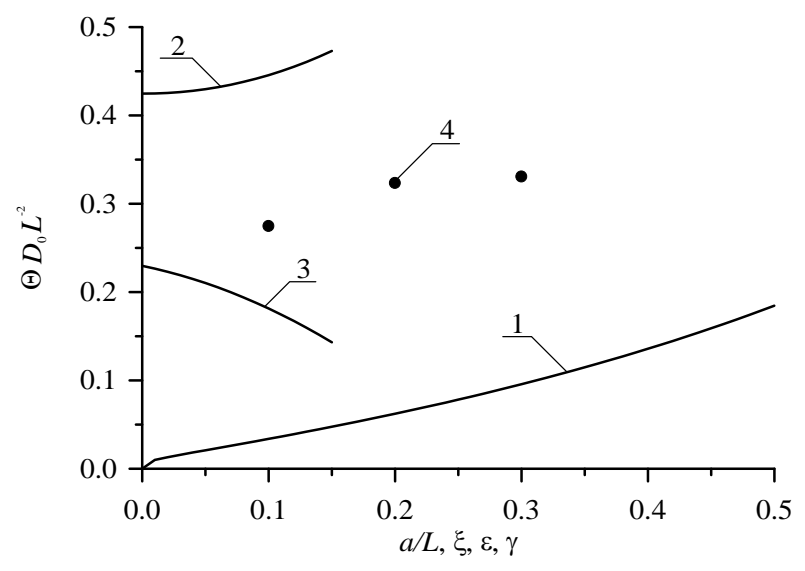

Fig.6. Dependences of dimensionless optimal annealing time of infused dopant.

Curve 1 describes dimensionless optimal annealing time as the function of the relation $a / L$ for $\xi=$ $\gamma=0$ and for equal to each other values of dopant diffusion coefficient in all parts of heterostructure. Curve 2 describes dimensionless optimal annealing time as the function of the parameter $\varepsilon$ for $a / L=1 / 2$ and $\xi=\gamma=0$ and for equal to each other values of dopant diffusion coefficient in all parts of heterostructure. Curve 3 describes dimensionless optimal annealing time as the function of the parameter $\xi$ for $a / L=1 / 2$ and $\varepsilon=\gamma=0$ and for equal to each other values of dopant diffusion coefficient in all parts of heterostructure. Curve 4 describes dimensionless optimal annealing time as the function of the parameter $\gamma$ for $a / L=1 / 2$ and $\varepsilon=\xi=0$ and for equal to each other values of dopant diffusion coefficient in all parts of heterostructure 


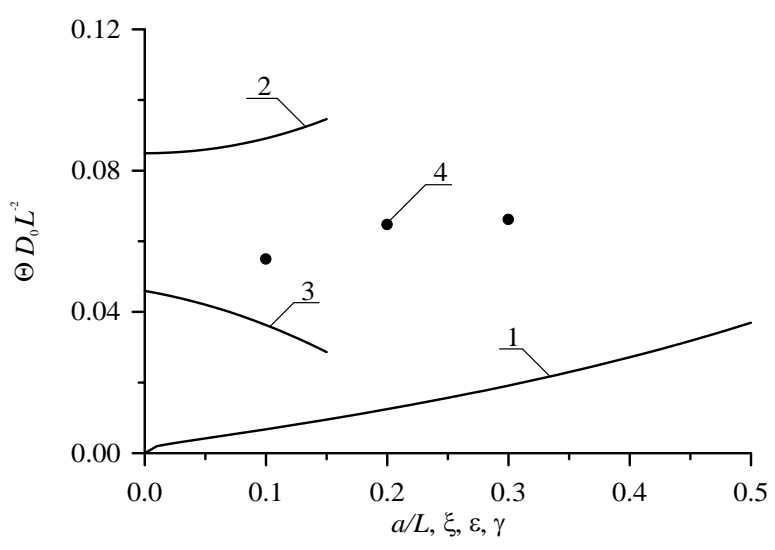

Fig.7. Dependences of dimensionless optimal annealing time of implanted dopant.

Curve 1 describes dimensionless optimal annealing time as the function of the relation $a / L$ for $\xi=$ $\gamma=0$ and for equal to each other values of dopant diffusion coefficient in all parts of heterostructure. Curve 2 describes dimensionless optimal annealing time as the function of the parameter $\varepsilon$ for $a / L=1 / 2$ and $\xi=\gamma=0$ and for equal to each other values of dopant diffusion coefficient in all parts of heterostructure. Curve 3 describes dimensionless optimal annealing time as the function of the parameter $\xi$ for $a / L=1 / 2$ and $\varepsilon=\gamma=0$ and for equal to each other values of dopant diffusion coefficient in all parts of heterostructure. Curve 4 describes dimensionless optimal annealing time as the function of the parameter $\gamma$ for $a / L=1 / 2$ and $\varepsilon=\xi=0$ and for equal to each other values of dopant diffusion coefficient in all parts of heterostructure

\section{ConClusions}

In this paper we analyzed variation of concentrations of infused and implanted dopants during manufacturing field-effect heterotransistors framework inverter circuit. We formulate recommendations for optimization annealing of dopant and/or radiation defects to decrease dimensions of considered transistors. We introduce an analytical approach to prognosis diffusive and ion types of doping. The approach gives a possibility to take into account at one time spatial and temporal variations of physical parameters of mass and heat transport and nonlinearity of both transports.

\section{ACKNOWLEDGEMENTS}

This work is supported by the agreement of August 27, 2013 № 02. .49.21.0003 between The Ministry of education and science of the Russian Federation and Lobachevsky State University of Nizhni Novgorod.

\section{REFERENCES}

[1] G. Volovich. Modern chips UM3Ch class D manufactured by firm MPS. Modern Electronics. Issue 2. P. 10-17 (2006).

[2] A. Kerentsev, V. Lanin, Constructive-technological features of MOSFET-transistors. Power Electronics. Issue 1. P. 34 (2008).

[3] A.O. Ageev, A.E. Belyaev, N.S. Boltovets, V.N. Ivanov, R.V. Konakova, Ya.Ya. Kudrik, P.M. Litvin, V.V. Milenin, A.V. Sachenko. Au-TiBx-n-6H-SiC Schottky barrier diodes: the features of current flow in rectifying and nonrectifying contacts. Semiconductors. Vol. 43 (7). P. 897-903 (2009).

[4] N.I. Volokobinskaya, I.N. Komarov, T.V. Matioukhina, V.I. Rechetniko, A.A. Rush, I.V. Falina, A.S. Yastrebov. Investigation of technological processes of manufacturing of the bipolar power high- 
voltage transistors with a grid of inclusions in the collector region. Semiconductors. Vol. 35 (8). P. 1013-1017 (2001).

[5] K.K. Ong, K.L. Pey, P.S. Lee, A.T.S. Wee, X.C. Wang, Y.F. Chong. Dopant distribution in the recrystallization transient at the maximum melt depth induced by laser annealing. Appl. Phys. Lett. 89 (17), 172111-172114 (2006).

[6] H.T. Wang, L.S. Tan, E. F. Chor. Pulsed laser annealing of Be-implanted GaN. J. Appl. Phys. 98 (9), 094901-094905 (2006).

[7] Yu.V. Bykov, A.G. Yeremeev, N.A. Zharova, I.V. Plotnikov, K.I. Rybakov, M.N. Drozdov, Yu.N. Drozdov, V.D. Skupov. Diffusion processes in semiconductor structures during microwave annealing. Radiophysics and Quantum Electronics. Vol. 43 (3). P. 836-843 (2003).

[8] V.V. Kozlivsky. Modification of semiconductors by proton beams (Nauka, Sant-Peterburg, 2003, in Russian).

[9] V.L. Vinetskiy, G.A. Kholodar', Radiative physics of semiconductors. ("Naukova Dumka", Kiev, 1979, in Russian).

[10] R. Gaska, M. Gaevski, R. Jain, J. Deng, M. Islam, G. Simin, M. Shur. Novel AlInN/GaN integrated circuits operating up to $500{ }^{\circ}$ C. Solid-State Electronics. Vol. 113. P. 22-27 (2015).

[11] E.L. Pankratov. Dopant diffusion dynamics and optimal diffusion time as influenced by diffusioncoefficient nonuniformity. Russian Microelectronics. Vol. 36 (1). P. 33-39 (2007).

[12] E.L. Pankratov. Redistribution of dopant during annealing of radiative defects in a multilayer structure by laser scans for production an implanted-junction rectifiers. Int. J. Nanoscience. Vol. 7 (4-5). P. 187-197 (2008).

[13] E.L. Pankratov. Decreasing of depth of implanted-junction rectifier in semiconductor heterostructure by optimized laser annealing. J. Comp. Theor. Nanoscience. Vol. 7 (1). P. 289-295 (2010).

[14] E.L. Pankratov, E.A. Bulaeva. Application of native inhomogeneities to increase compactness of vertical field-effect transistors. J. Comp. Theor. Nanoscience. Vol. 10 (4). P. 888-893 (2013).

[15] E.L. Pankratov, E.A. Bulaeva. An approach to manufacture of bipolar transistors in thin film structures. On the method of optimization. Int. J. Micro-Nano Scale Transp. Vol. 4 (1). P. 17-31 (2014).

[16] E.L. Pankratov, E.A. Bulaeva. Increasing of sharpness of diffusion-junction heterorectifier by using radiation processing. Int. J. Nanoscience. Vol. 11 (5). P. 1250028-1250035 (2012).

[17] Z.Yu. Gotra. Technology of microelectronic devices (Radio and communication, Moscow, 1991).

[18] P.M. Fahey, P.B. Griffin, J.D. Plummer. Point defects and dopant diffusion in silicon. Rev. Mod. Phys. V. 61. № 2. P. 289-388 (1989).

[19] Yu.D. Sokolov. About the definition of dynamic forces in the mine lifting. Applied Mechanics. Vol. 1 (1). P. 23-35 (1955).

[20] E.L. Pankratov. Dynamics of delta-dopant redistribution during heterostructure growth. The Eur. Phys. J. B. Vol. 57 (3). P. 251-256 (2007).

\section{AUTHORS}

Pankratov Evgeny Leonidovich is a Doctor of Science, an Associate Professor of Nizhny Novgorod State University. He has 189 published papers in area of his researches.

Bulaeva Elena Alexeevna is a Master of Science, a PhD student of Nizhny Novgorod State University. She has 138 published papers in area of his researches. 